\title{
A halo mass-concentration relation from weak lensing
}

\author{
Rachel Mandelbaum ${ }^{1}$, Uroš Seljak ${ }^{2,3}$ and \\ Christopher M Hirata ${ }^{4}$ \\ ${ }^{1}$ Institute for Advanced Study, Einstein Drive, Princeton, NJ 08540, USA \\ ${ }^{2}$ Institute for Theoretical Physics, University of Zurich, Zurich, Switzerland \\ ${ }^{3}$ Department of Physics, University of California, Berkeley, CA 94720, USA \\ ${ }^{4}$ Mail Code 130-33, Caltech, Pasadena, CA 91125, USA \\ E-mail:rmandelb@ias.edu, seljak@physik.unizh.ch and \\ chirata@tapir.caltech.edu
}

Received 16 May 2008

Accepted 7 July 2008

Published 6 August 2008

Online at stacks.iop.org/JCAP $/ 2008 / \mathrm{i}=08 / \mathrm{a}=006$

doi:10.1088/1475-7516/2008/08/006

\begin{abstract}
We perform a statistical weak lensing analysis of dark matter profiles around tracers of halo mass from galaxy-size to cluster-size halos. In this analysis we use 170640 isolated $\sim L_{*}$ galaxies split into ellipticals and spirals, 38236 groups traced via isolated spectroscopic luminous red galaxies and 13823 maxBCG clusters from the Sloan Digital Sky Survey covering a wide range of richness. Together these three samples allow a determination of the density profiles of dark matter halos over three orders of magnitude in mass, from $10^{12} M_{\odot}$ to $10^{15} M_{\odot}$. The resulting lensing signal is consistent with a Navarro-Frenk-White (NFW) or Einasto profile on scales outside the central region. In the inner regions, uncertainty in modeling of the proper identification of the halo center and inclusion of baryonic effects from the central galaxy make the comparison less reliable. We find that the NFW concentration parameter $c_{200 b}$ decreases with halo mass, from around 10 for galactic halos to 4 for cluster halos. Assuming its dependence on halo mass in the form of $c_{200 b}=c_{0}\left(M / 10^{14} h^{-1} M_{\odot}\right)^{-\beta}$ we find $c_{0}=4.6 \pm 0.7$ (at $z=0.22$ ) and $\beta=0.13 \pm 0.07$, with very similar results for the Einasto profile. The slope $(\beta)$ is in agreement with theoretical predictions, while the amplitude is about two standard deviations below the predictions for this mass and redshift, but we note that the published values in the literature differ at a level of $10-20 \%$ and that for a proper comparison our analysis should be repeated in simulations. We compare our results to other recent determinations, some of which find significantly higher concentrations. We
\end{abstract}


discuss the implications of our results for the baryonic effects on the shear power spectrum: since these are expected to increase the halo concentration, the fact that we see no evidence of high concentrations on scales above $20 \%$ of the virial radius suggests that baryonic effects are limited to small scales, and are not a significant source of uncertainty for the current weak lensing measurements of the dark matter power spectrum.

Keywords: dark matter, structure of galaxies, gravitational lensing

ArXiv ePrint: 0805.2552

\section{Contents}

1. Introduction 2

2. Data and analysis 5

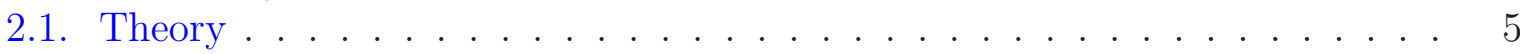

2.2. Data . . . . . . . . . . . . . . . . . . 8

2.2.1. MaxBCG cluster lenses. . . . . . . . . . . . . . . . 8

2.2.2. Spectroscopic LRGs. . . . . . . . . . . . . . . . . . . . . . . 99 9

2.2.3. $L_{*}$ lenses. . . . . . . . . . . . . . . . . . . 9

2.2.4. Lensing sources. . . . . . . . . . . . . . . . . . 11

2.3. Analysis . . . . . . . . . . . . . . . . . . . . . 11

3. Results: lensing signal and fits to NFW profile $\quad 12$

3.1. Description of fits . . . . . . . . . . . . . . . . 13

3.1.1. Basic fits. . . . . . . . . . . . . . . . . . 14

3.1.2. Fits with offsets. . . . . . . . . . . . . . . 16

3.1.3. Fits with fixed power law scaling. . . . . . . . . . . . . . . 17

3.1.4. Systematic tests of fitting procedure. . . . . . . . . . . . . . . 18

3.2. Concentration-mass relation . . . . . . . . . . . . . . 18

3.3. Comparison against previous observations . . . . . . . . . . . . . . . 19

3.4. Comparison to simulations . . . . . . . . . . . . . . . . . 21

3.5. Implications for shear-shear lensing . . . . . . . . . . . . . . . 22

4. Conclusions 23

Acknowledgments $\quad 24$

$\begin{array}{ll}\text { References } & 24\end{array}$

\section{Introduction}

The density profile of dark matter (DM) halos is one of the fundamental predictions of cosmological models in the non-linear regime, determined using $N$-body simulations [1][15]. The profile is often parameterized with the so called NFW profile [12], a broken power law characterized by a scale radius where the slope is approximately -2 , and by the virial radius which parameterizes its mass. The ratio of the latter to the former is called the concentration $c$, and is a measure of the density of the halo in the inner regions: 
a higher concentration implies a higher density of the halo at a fixed fraction of virial radius. The concentration is predicted to be mildly dependent on mass such that higher mass halos are less concentrated than lower mass halos. Because the normalization of the concentration-mass relation depends on the matter power spectrum normalization, its shape, and the matter density $\Omega_{\mathrm{m}}$, measuring the halo profile as a function of halo mass can teach us about the underlying cosmology.

Many different methods have been used to measure the halo profile of clusters. Kinematic tracers such as satellite galaxies, in combination with a Jeans analysis or caustics analysis, can give information over a wide range of physical scales and halo masses. While the issues of relaxation, velocity bias, anisotropy of the orbits and interlopers continue to be debated and need to be carefully addressed, recent results suggest a good agreement with theoretical predictions [16]-[21]. Hydrostatic analyses of $\mathrm{x}$-ray intensity profiles of clusters use x-ray intensity and temperature as a function of radius to reconstruct the density profile. They have the benefit of thermal gas pressure being isotropic, but may be biased due to the possible presence of other sources of pressure support, such as turbulence, cosmic rays or magnetic fields. These cannot be strongly constrained for typical clusters with present x-ray data [22], but could modify the hydrostatic equilibrium and affect the conclusions of such analyses. Recent results are encouraging and are in a broad agreement with predictions, although most require concentrations that are higher than those predicted by a concordance cosmology [23]-[25]. While the above-mentioned systematic biases cannot be excluded, the small discrepancy could also be due to baryonic effects in the central regions, due to selection of relaxed clusters that may be more concentrated than average [25], or due to the fact that at a given x-ray flux limit, the more concentrated clusters near the limiting mass are more likely to be included in the sample [26].

Gravitational lensing is by definition sensitive to the total mass, and therefore yields one of the most promising methods for measuring the mass profile. Some analyses have combined strong and weak lensing or velocity dispersion constraints for individual clusters [27]-[32] to derive the profile, and in some cases concentrations have been found above the predictions from simulations. However, strong lensing is affected by the mass distribution in the very inner parts of the cluster, and both ellipticity of matter and stars from the central galaxy have a significant effect on the strong lensing signatures, so these analyses are not necessarily measuring the primordial dark matter halo profile [33]. Furthermore, both strong lensing and x-ray analyses are susceptible to selection bias effects when changing the DM concentration at fixed mass [26]. The problems in interpreting the observations on small scales, where baryons play an important role, suggest that we should focus on larger scales if we want to compare observations to theoretical predictions from $N$-body simulations. Weak lensing is arguably the most promising tool that can be used for measuring the profile out to scales of several $h^{-1} \mathrm{Mpc}$. It has the advantage that outside the central region, the dark matter distribution is likely to be unaffected by baryons, so a comparison against $N$-body simulations should be more reliable.

Many previous weak lensing analyses have focused on individual clusters (for example, [34,35]). Measuring the matter distribution of individual clusters has its advantages, since it allows a comparison with the light and gas distributions on an individual basis, and so can constrain models that relate the two, such as MOND 
versus CDM [36]. However, since lensing measures a projected surface density with a window that extends hundreds of megaparsecs away, other mass perturbation along the line of sight will also produce a lensing signal and thus act as a source of noise when extracting the cluster density profile. Some of these structures may be correlated with the cluster itself, for example those that are falling into the cluster along the filaments connected to the cluster [37], and can be defined as part of the cluster profile, while other structures may be completely unrelated mass concentrations tens or hundreds of megaparsecs away [38].

The measurement of the dark matter profile can therefore be quite noisy for individual clusters. Stacking the signal from many clusters can ameliorate this problem, since only the mass density correlated with the cluster will produce a signal. In this way, the measurement determines the true average cluster-mass profile in the same way as was defined in simulations. Such a statistical approach is thus advantageous if one is to compare the observations to theoretical predictions, which also average over a large number of halos in simulations. In fact, simulations show a significant scatter in the shapes of individual halo profiles [2,39], so stacking many halos will reduce the fluctuations due to noise caused by uncorrelated structures along the line of sight, due to shape measurement noise, and due to the shape variations of individual halos. A final advantage of stacking is that it allows for the lensing measurement of lower mass halos, where individual detection is impossible due to their lower shears relative to clusters. Individual high signal-tonoise cluster observations and those based on stacked analysis of many clusters are thus complementary to each other at the high mass end, with the stacked analysis drastically increasing the available baseline in mass.

The statistical approach based on stacked clusters has been applied to a small number of clusters before [40,41]. The Sloan Digital Sky Survey (SDSS) provides an ideal data set for such analysis: it covers a significant fraction of the sky containing $\sim 10^{4}$ clusters up to $z \sim 0.3$, providing a large volume of the universe over which the clusters can be observed. The SDSS spectroscopy and multicolor imaging enables precise redshift determination, so we can determine the profile as a function of true transverse separation rather than angle. In a previous analysis, we have used a sample of 43335 groups and clusters as traced via luminous red galaxies (LRGs) [42] to derive average mass density profiles of groups with masses between $3 \times 10^{13} h^{-1} M_{\odot}$ and $1.3 \times 10^{14} h^{-1} M_{\odot}$. We have also performed a halo model analysis of the lensing signal of isolated $\sim L_{*}$ elliptical galaxies in [43] to ensure that they are all in the field, and will augment that sample with isolated $\sim L_{*}$ spiral galaxies. In this paper we extend these previous analyses to the new sample of 13823 maxBCG clusters presented in [44], which extends the mass range to $\sim 6 \times 10^{14} h^{-1} M_{\odot}$. We also compare our results against an independent analysis of these clusters in [45], though both analyses have included objects other than the public maxBCG catalog selected in different ways. We then combine the elliptical, spiral, LRG and maxBCG analyses to obtain information about the halo density profile over a wide range of masses, and compare them to theoretical predictions from $N$-body simulations.

We begin in section 2 by presenting the theory behind our measurement, the data, and the analysis method used. The results are presented in section 3, including a discussion of how to compare them with other observations and with theory. Our conclusions derived from this analysis and comparison with theory are given in section 4 . 


\section{Data and analysis}

\subsection{Theory}

We follow the same methodology as in [42], so we refer the reader to that paper for a more detailed description of the analysis process. In brief, cluster-galaxy and galaxy-galaxy weak lensing provide a simple way to probe the connection between clusters (or galaxies) and matter via their cross-correlation functions $\xi_{\mathrm{cl}, \mathrm{m}}(\vec{r})\left(\right.$ or $\left.\xi_{\mathrm{g}, \mathrm{m}}(\vec{r})\right)$, which can be related to the projected surface density

$$
\Sigma(R)=\bar{\rho} \int\left[1+\xi_{\mathrm{cl}, \mathrm{m}}\left(\sqrt{R^{2}+\chi^{2}}\right)\right] \mathrm{d} \chi
$$

where $R$ is the transverse separation and $\chi$ the radial direction over which we are projecting. We are ignoring the effects from the radial window, which is hundreds of megaparsecs broad and not relevant at cluster scales. The surface density is then related to the observable quantity for lensing, the differential surface density,

$$
\Delta \Sigma(R)=\gamma_{\mathrm{t}}(R) \Sigma_{\mathrm{c}}=\bar{\Sigma}(<R)-\Sigma(R),
$$

where the second relation is true only in the weak lensing limit, for a matter distribution that is axisymmetric along the line of sight (which is naturally achieved by our procedure of stacking thousands of clusters to determine their average lensing signal). This observable quantity can be expressed as the product of two factors, a tangential shear $\gamma_{\mathrm{t}}$ and a geometric factor:

$$
\Sigma_{\mathrm{c}}=\frac{c^{2}}{4 \pi G} \frac{D_{\mathrm{S}}}{D_{\mathrm{L}} D_{\mathrm{LS}}\left(1+z_{\mathrm{L}}\right)^{2}},
$$

where $D_{\mathrm{L}}$ and $D_{\mathrm{S}}$ are angular diameter distances to the lens and source, and $D_{\mathrm{LS}}$ is the angular diameter distance between the lens and source. Unless otherwise noted, all computations assume a flat $\Lambda \mathrm{CDM}$ universe with $\Omega_{\mathrm{m}}=0.27$ and $\Omega_{\Lambda}=0.73$. Distances quoted for transverse lens-source separation are comoving (rather than physical) $h^{-1} \mathrm{kpc}$, where $H_{0}=100 \mathrm{~h} \mathrm{~km} \mathrm{~s}^{-1} \mathrm{Mpc}^{-1}$. Likewise, the differential surface density $\Delta \Sigma$ is computed in comoving coordinates, and the factor of $\left(1+z_{\mathrm{L}}\right)^{-2}$ arises due to our use of comoving coordinates.

For this paper, we are primarily interested in the contribution to the cluster-mass or galaxy-mass cross-correlation from the halo profile itself, rather than from neighboring halos (halo-halo term), and hence

$$
\Sigma(R)=\int_{-\infty}^{\infty} \rho\left(r=\sqrt{\chi^{2}+R^{2}}\right) \mathrm{d} \chi
$$

The halo-halo term for clusters in host halos can be modeled simply using the clusterdark matter or galaxy-dark matter cross-power spectrum as in, e.g., [46], and is only important for $R>\sim 2 h^{-1}$ Mpc. Nevertheless, we compute this component and include it in the model as a fixed term which we obtain by computing first the mass of the clusters, deriving the corresponding halo bias using the bias-mass relation $[47,48]$ and using the linear power spectrum multiplied with the halo bias to obtain the halo-halo term. This procedure has been shown to work well in comparison to simulations [46], so we use the 
bias-mass relation from that paper but with $\sigma_{8}=0.75$ and $\Omega_{\mathrm{m}}=0.27$, computed at the mean redshift of each sample.

We can model the one-halo term for each sample as a sum of the stellar component, only important on scales below $\sim 100 h^{-1} \mathrm{kpc}$, and an NFW dark matter profile [12]:

$$
\rho(r)=\frac{\rho_{s}}{\left(r / r_{s}\right)\left(1+r / r_{s}\right)^{2}} \text {. }
$$

It is convenient to reparameterize it using two parameters, concentration $c=r_{\text {vir }} / r_{s}$ and virial mass $M$. The virial radius $r_{\text {vir }}$ and $\rho_{s}$ can be related to $M$ via consistency relations. The first is that the virial radius is that within which the average density is equal to 200 times the mean density:

$$
M_{200 b}=\frac{4 \pi}{3} r_{\text {vir }}^{3}(200 \bar{\rho}) \text {. }
$$

The second relation, used to determine $\rho_{s}$ from $M$ and $c$, is simply that the volume integral of the density profile to the virial radius must equal the virial mass. The NFW concentration $c$ is a weakly decreasing function of halo mass, with a typical dependence as

$$
c_{200 b}=\frac{c_{0}}{1+z}\left(\frac{M}{M_{0}}\right)^{-\beta},
$$

with $\beta \sim 0.1[2,39,49]$, making this profile a one-parameter family of profiles. The normalization depends on the non-linear mass, which is the mass within spheres in which the rms fluctuation in the linear regime is 1.68; for the typical range of cosmological models, one expects $c \approx 5-6$ at $M_{0}=10^{14} h^{-1} M_{\odot}$.

As an example of how the lensing signal varies with concentration, in figure 1 we show the predicted NFW lensing signal as a function of transverse separation for $M_{200 b}=10^{12}$ (galaxy scale) and $10^{14} h^{-1} M_{\odot}$ (cluster scale) halos, for several plausible concentration values. The vertical line shows the minimum scale used for our fits, and it is clear that the lensing signal above those scales can differentiate between different concentration values. This discriminating power stems in part from the fact that the lensing signal reflects the differential surface density, which draws information from smaller scales to larger scales.

We fit the data to the model assuming a spherical NFW profile. In [46], it was shown that spherical NFW profiles do an excellent job of describing the stacked lensing signal from simulations for a variety of masses, with the masses and concentrations of the best-fit profiles related to the real masses and concentrations in the simulations in a particular way (to be discussed further below). The fit $\chi^{2}$ values were very good when the error bars used on the simulated lensing signal were a tenth of our current error bars, so henceforth we consider only spherical NFW fits rather than trying to account for the averaging of triaxial halos.

Although we could include the effects of the central galaxy to make the model accurate on scales below $20 \%$ of the virial radius, there are reasons why we should exclude this information from the fits if we want to compare against $N$-body simulations. First, the baryonic effect that we assume may not be completely accurate because of uncertainties in the stellar mass to light ratio, and in the dark matter response to the presence of baryons and stars forming out of them. The latter is often modeled as adiabatic contraction $[50,51]$, but this prescription may inaccurately describe the actual effect, 


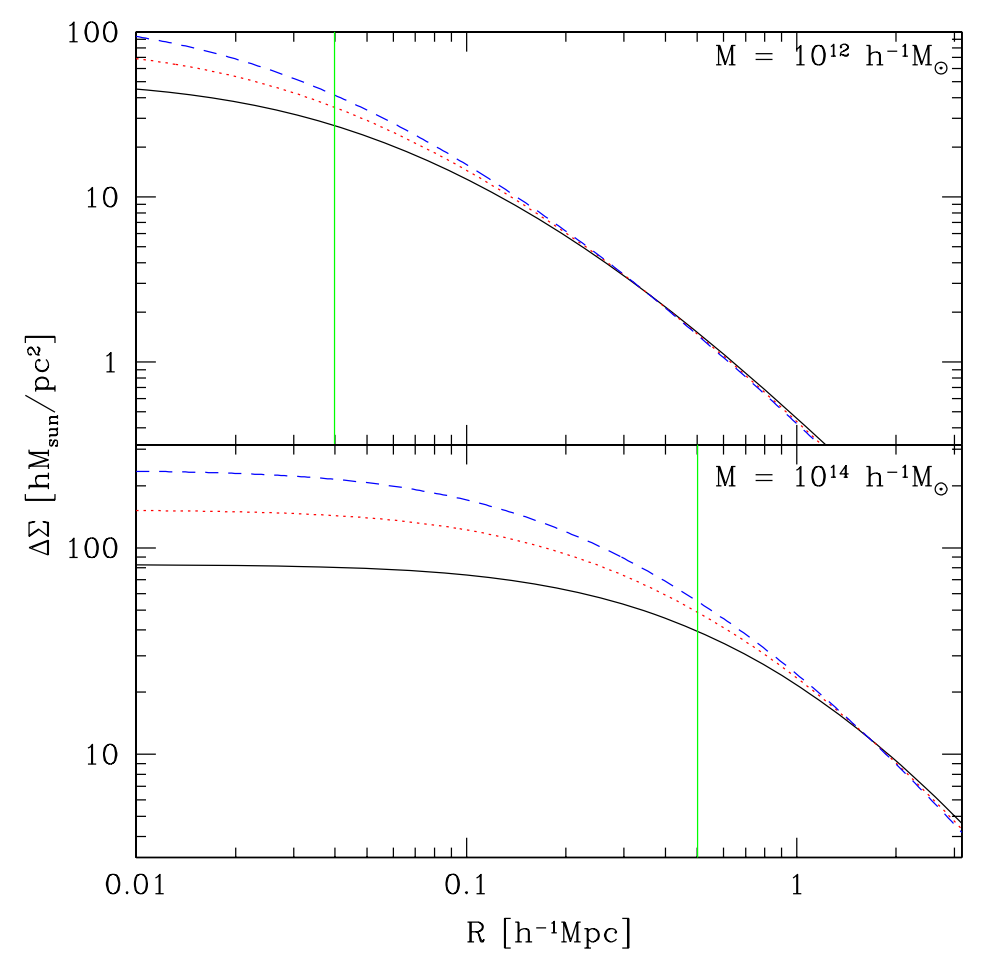

Figure 1. The NFW profile theoretical lensing signal $\Delta \Sigma$ for two mass scales. In the upper plot, with $M=10^{12} h^{-1} M_{\odot}$, the concentration is varied from 7 to 10 to 13 . In the lower panel, with $M=10^{14} h^{-1} M_{\odot}$, it is varied from 3 to 5 to 7 . The curves on each panel are normalized to the same virial mass $M_{200 b}$. Vertical lines show the minimum scale for our fits.

depending on the formation history of the galaxy, group, or cluster in question [52]. The second, possibly more important effect is that for cluster samples such as maxBCG, the cluster center cannot always be reliably determined using the optical information. This uncertainty has two causes: first, that a brightest cluster galaxy (BCG) may not be located at the deepest part of the cluster potential, but may be offset from it due to (for example) perturbations from infalling satellites; and second (and more importantly), that the maxBCG algorithm may choose the wrong BCG. Studies comparing the BCG position to the center defined by either x-ray intensity or by average satellite velocity have found that the typical displacement is about $2-3 \%$ of the virial radius when the BCG is properly identified [44], [53]-[55]. The last of these studies finds that for about $10 \%$ of BCGs, the displacement extends to above $10 \%$ of the virial radius. It is interesting to note that selecting blue-core BCGs significantly reduces the displacement to below $1 \%$ of the virial radius [55]. However, while the multi-band SDSS aperture photometry could be used to select blue-core BCGs, we do not undertake this approach for this analysis because of decreased $S / N$.

Another study that includes red galaxy photometric errors (i.e., both effects rather than just the first) finds that the median displacement is $10 \%$ of the virial radius [56]. If the assumed center is displaced from the true center, then this can have a significant effect on the density distribution in the inner parts, mimicking a halo profile with a lower 
concentration $[57,58]$, while in the outer parts the effect is significantly smaller. To avoid this problem, we use a minimum scale for the fits of $500 \mathrm{~h}^{-1} \mathrm{kpc}$ for the maxBCG clusters (typically half the virial radius), and present evidence that this approach is robust to the effects of centroiding problems, at the expense of decreased statistical power.

We note that this problem has also been addressed in a statistical manner, using mock catalogs to determine both the fraction of clusters affected by centroiding problems, and also the distribution of projected offsets from the true cluster center for those that are centroided wrong [45]. Using this correction, one can in principle correct for the effect and fit to significantly smaller scales; however the correction is quite dependent on the content of the mock catalogs, and any deviation from reality may invalidate it. For example, the mock catalogs suggest that misidentification of the center is more of a problem at lower halo masses, while our visual inspection of clusters suggests the opposite, in the sense that the most massive clusters (with richness $N_{200}>80$ ) have significant bimodality and substructure, as expected from a hierarchical cosmological model where the most massive clusters formed the latest. As a result, we exclude these most massive clusters from the analysis entirely. For the remaining sample, we choose the different approach of avoiding the inner parts of the cluster (where the effect is strongest), thereby decreasing the systematic error at the expense of an increase in statistical error.

\subsection{Data}

The data used here are obtained from the SDSS [59], an ongoing survey to image roughly $\pi$ steradians of the sky, and follow up approximately one million of the detected objects spectroscopically [60]-[62]. The imaging is carried out by drift-scanning the sky in photometric conditions [63,64], in five bands (ugriz) [65,66] using a specially designed wide-field camera [67]. These imaging data are used to create the source catalog that we use in this paper. In addition, objects are targeted for spectroscopy using these data [68] and are observed with a 640-fiber spectrograph on the same telescope [69]. All of the data are processed by completely automated pipelines that detect and measure photometric properties of objects, and astrometrically calibrate the data [70]-[72]. The SDSS has had seven major data releases [73]-[80].

In the subsections that follow, we describe the lens and source samples.

2.2.1. MaxBCG cluster lenses. Our highest mass lens sample consists of 13823 maxBCG clusters $[44,53]$, which are identified by the concentration of galaxies in color-position space, using the well known red galaxy color-redshift relation [81]. The sample is based on 7500 square degree of imaging data in SDSS. There is a tight mass-richness relation that has been established using dynamical information across a broad range of halo mass [82]. This is also confirmed with the lensing analysis, as shown below and in $[45,83,84]$.

The redshift range of the maxBCG catalog is $0.1<z<0.3$, and the upper cutoff ensures that the lenses still have a sufficient number of sources behind them. Within these redshift limits, the sample is approximately volume-limited with a number density of $3 \times 10^{-5} \mathrm{~h}^{3} \mathrm{Mpc}^{-3}$, except for a tendency towards higher number density at the lower end of this redshift range [84]. The main tracers of halo mass provided by the maxBCG team are a rescaled richness $N_{200}$ (number of red galaxies above $0.4 L_{*}$ ), total luminosity $L_{200}$ (the luminosity in those red galaxies, including the BCG) and BCG luminosity $L_{\mathrm{BCG}}$. 
In this paper we use richness $N_{200}$ as a primary tracer of halo mass. However, we observe (based on [84]) that the lower mass end of the public catalog overlaps with the LRG samples used for this work, so we eliminate those clusters with the lowest richness, $N_{200}=10$ and 11 ( $1 / 3$ of the public catalog), and split the remainder into six narrow $N_{200}$ bins as shown in table 1 (except for those with $N_{200}>80$, as described in section 2.1). The widest of these spans a factor of 1.4 in $N_{200}$, so if we assume that the mass is proportional to this observable, then the mass bin is a factor of 1.4 wide without any mass-observable scatter. In reality such scatter exists, but even a factor of two scatter in the mass at fixed $N_{200}$ would give a mass distribution less than an order of magnitude wide. As we have shown in [46], when fitting for a single halo mass on a stacked sample that is less than an order of magnitude wide, the best-fit mass is a good proxy (within $~ 10 \%$ ) for the true mean mass of the sample.

2.2.2. Spectroscopic LRGs. For the next sample with lower average halo mass, we use the spectroscopic luminous red galaxy (LRG) sample [60], including area beyond Data Release 4 (DR4). The total area coverage for this spectroscopic sample is 5154 square degrees, as available in the NYU Value Added Galaxy Catalog (VAGC [85]) at the time of the original publication of our lensing work with this sample [42].

For the LRG sample, we define luminosities using $r$-band model magnitudes, extinction-corrected using reddening maps from [86]. We apply a $k+e$-correction (combined $k$-correction and correction for evolution of the spectrum) to redshift zero using stellar population synthesis code from [87]; this magnitude is denoted as $M_{0.0_{r}}$ to distinguish it from the magnitudes used for the main sample, which are corrected to $z=0.1$. In our original work, the LRGs were split into two luminosity bins, $M_{0.0_{r}} \geq-22.3$ and $M_{0.0_{r}}<-22.3$. To reduce the overlap between the LRGs and the cluster samples to $<5 \%$, we use the fainter bin in its entirety but only use the $-22.6 \leq M_{0.0_{r}}<-22.3$ subset of the brighter sample.

These LRG samples were derived from the full LRG sample after eliminating $15 \%$ of the sample using a cylindrical density estimator, designed to avoid satellite galaxies due to their extra lensing signal from the host halo. Specifically, the LRGs were each required to be the only or the brightest LRG in a cylinder with radius $R=2 h^{-1} \mathrm{Mpc}$ and line-of-sight separation $\delta v= \pm 1200 \mathrm{~km} \mathrm{~s}^{-1}$. This cut is conservative, in the sense that for typical groups and low mass clusters, we may have excluded some host galaxies; however, the host sample purity is sufficiently important for this analysis that we tend towards the conservative side. More information about the LRG samples is available in table 1.

2.2.3. $L_{*}$ lenses. Finally, we include lower luminosity samples from [43] that have been shown with a robust environment estimator to consist of field galaxies. For those samples, we used galactic extinction-corrected $r$-band Petrosian magnitudes, $k$-corrected to $z=0.1$ using KCORRECT V4_1_4 [88], denoted $M_{0.1_{r}}$.

The samples used here correspond to L3, L4, and L5faint isolated ellipticals from [43], where the isolated ellipticals are the half of the elliptical sample at those luminosities determined to be in the field using a cylindrical density estimator. We also include a sample not shown there but from the same data, of L3 and L4 isolated spirals, or $85 \%$ of the spirals in those luminosity bins. For reference, $L_{*}$ is within L4, so our samples range from slightly below to slightly above $L_{*}$. In this context, the elliptical and spiral 
Table 1. Summary of all lens samples used in this paper. Note that observable definitions (such as luminosity) are not necessarily the same for each parent sample; see the relevant subsection for details. Additional sample cuts are indicated by the parent samples, which typically are defined using flux and color cuts.

\begin{tabular}{|c|c|c|c|c|c|c|}
\hline Parent sample & Isolation method & Observable cut & Redshift cut & $\langle z\rangle$ & $N$ & Name \\
\hline MaxBCG clusters & MaxBCG method & $\begin{array}{l}12 \leq N_{200} \leq 13 \\
14 \leq N_{200} \leq 19 \\
20 \leq N_{200} \leq 28 \\
29 \leq N_{200} \leq 39 \\
40 \leq N_{200} \leq 54 \\
55 \leq N_{200} \leq 79\end{array}$ & $0.1<z<0.3$ & 0.22 & $\begin{array}{r}2531 \\
3372 \\
1618 \\
614 \\
248 \\
109\end{array}$ & \\
\hline Spectroscopic LRGs & Cylindrical & $\begin{array}{l}M_{0.0_{r}}>-22.3 \\
-22.3 \geq M_{0.0_{r}}>-22.6\end{array}$ & $0.15<z<0.35$ & 0.24 & $\begin{array}{l}27700 \\
10536\end{array}$ & \\
\hline Main spectroscopic & Cylindrical & $\begin{array}{l}-20 \leq M_{0.1_{r}}<-19, \text { frac_deV } \geq 0.5 \\
-21 \leq M_{0.1_{r}}<-20, \text { frac_deV } \geq 0.5 \\
-21.5 \leq M_{0.1_{r}}<-21, \text { frac_deV } \geq 0.5 \\
-20 \leq M_{0.1_{r}}<-19, \text { frac_deV }<0.5 \\
-21 \leq M_{0.1_{r}}<-20, \text { frac_deV }<0.5\end{array}$ & $z>0.02$ & $\begin{array}{l}0.07 \\
0.10 \\
0.13 \\
0.07 \\
0.10\end{array}$ & $\begin{array}{l}20150 \\
46130 \\
23485 \\
38640 \\
42235\end{array}$ & $\begin{array}{l}\text { L3 elliptical } \\
\text { L4 elliptical } \\
\text { L5 faint elliptical } \\
\text { L3 spiral } \\
\text { L4 spiral }\end{array}$ \\
\hline
\end{tabular}


samples are chosen using the SDSS frac_deV parameter, which determines whether the light profile is closer to a de Vaucouleurs or exponential profile. More details of these five lens samples are shown in table 1.

We do not use the fainter samples (L1 and L2) from previous work because the detection significance of the weak lensing signal is low, so they cannot constrain the halo concentration. We also avoid the brighter elliptical samples because they overlap with the spectroscopic LRGs, and the brighter spiral samples because they are nearly empty.

2.2.4. Lensing sources. The source sample used is the same as that originally described in [89]. This source sample includes over 30 million galaxies from the SDSS imaging data with $r$-band model magnitude brighter than 21.8, with shape measurements obtained using the REGLENS pipeline, including PSF correction done via re-Gaussianization [90] and with cuts designed to avoid various shear calibration biases. In addition, there are also uncertainties due to photometric redshifts and/or redshift distributions of background galaxies, which were originally calibrated using DEEP2 Groth strip data, as well as due to other issues affecting the calibration of the lensing signal, such as the sky subtraction uncertainties, intrinsic alignments, magnification bias, star-galaxy separation, and seeingdependent systematics. The overall $1 \sigma$ calibration uncertainty was estimated to be eight per cent [89], though the redshift calibration component of this systematic error budget has recently been significantly decreased due to the availability of more spectroscopic data [91]. The calibration mainly affects the mass estimation rather than the derived density profiles, so it is not of significant concern for this paper due to the weak dependence of concentration on mass.

An additional concern is the relative calibration of lensing measurements from different lens samples that were published in different papers. If there are calibration differences between these measurements, then the power law scaling of the $c(M)$ relation might be misestimated. However, it seems unlikely that there can be significant calibration differences between the different measurements, for two reasons. First, the same version of the source catalog was used for each one. This suggests that any shear calibration or star/galaxy separation issues are the same for each measurement. There may be very slight variation due to the different mean lens redshifts, which changes the effective mean redshift of the sources, but our previous tests of the source catalog for relative shear calibration as a function of apparent magnitude and size [89] rule out changes in the calibration of the shear that are significant relative to the $1 \sigma$ statistical error on these measurements. Second, we have rigorously tested the calibration of the source redshift distributions using the zCOSMOS and DEEP2 spectroscopic samples, and found that the calibrations for the lens redshift distributions used here are the same within several per cent, which is again smaller than the $1 \sigma$ measurement error on the lensing signals used here [91]. In short, while there is some small uncertainty (discussed above) in the absolute lensing signal calibration, we have little reason to believe there is any significant discrepancy between the calibrations for the different lens subsamples.

\subsection{Analysis}

Calculation of the signal is described in detail in [42]. Briefly, we compute the weights based on noise and redshift information for each lens-source pair, summing them using a 
minimal variance estimator. We compute the signal around random points and subtract it from the signal around real lenses to eliminate contributions from systematic shear. The signal must be boosted, i.e. multiplied by $B(R)=n(R) / n_{\text {rand }}(R)$, the ratio of the number density of sources relative to the number around random points, in order to account for dilution by sources that are physically associated with lenses, and therefore not lensed. The former correction is only important on scales above those used in this paper $\left(>5 h^{-1} \mathrm{Mpc}\right)$ and the latter on scales below $20 \%$ of the virial radius, which we do not use for the fits in this paper. To determine errors on the lensing signal, we divide the survey area into 200 bootstrap subregions, and generate 2500 bootstrap-resampled data sets. We note that the effects of non-weak shear, magnification bias, sky subtraction and intrinsic alignments, discussed in more detail in [42,89], are negligible on the scales used in this paper.

The errors determined from the bootstrap are used in plots of the signal with errors; however, the bootstrap covariance matrices can be quite noisy and therefore inappropriate to use for weighting in the $\chi^{2}$ minimization fitting. To avoid this problem, we determine analytic, diagonal covariance matrices (including shape noise), which we have shown in [89] to be a less noisy version of the bootstrap covariance matrices with agreement in size of the errors at the $\sim 10 \%$ level. These covariance matrices, which are far less noisy, are used to perform the fits on each bootstrap-resampled data set. The distributions of output parameters from all the bootstrap-resampled data sets are used to determine errors on the fit parameters.

The fits are for eleven parameters, using the lensing signal from the thirteen lens samples in table 1.

- The normalization and slope of the concentration-mass relation, equation (7) (two parameters).

- The normalization and slope of the relation between mass $M_{200 b}$ and maxBCG richness $N_{200}$ (two parameters):

$$
M_{200 b}=M_{0}\left(\frac{N_{200}}{20}\right)^{\gamma} .
$$

- The masses of the two LRG and five lower luminosity samples (7 parameters).

As shown in equation (7), the concentration is expected to scale with redshift. Since the samples are at different mean redshifts, we use the expected redshift scaling to fit for a normalization $c_{0}(z=0.22)$ (the mean, lensing-weighted redshift of the maxBCG sample).

\section{Results: lensing signal and fits to NFW profile}

We perform the analysis described in section 2.3 on the lensing signal $\Delta \Sigma$ for all the lens samples. Figure 2 shows the lensing signal $\Delta \Sigma$ with the best-fit model in the joint fits for $c_{200 b}\left(M_{200 b}, z=0.22\right)$, for $M_{200 b}\left(N_{200}\right)$ for the maxBCG clusters, and best-fit masses for the spectroscopic LRGs and the lower luminosity galaxy samples. Figure 3 shows the signal and best-fit model for the lower luminosity samples, and figure 4 shows the same for the two LRG samples. We see that for the maxBCG sample there is a strong lensing signal over the entire range of richness, and the lensing signal is increasing with richness as expected. The best-fit parameters for concentration and mass (to be described below) 
A halo mass-concentration relation from weak lensing

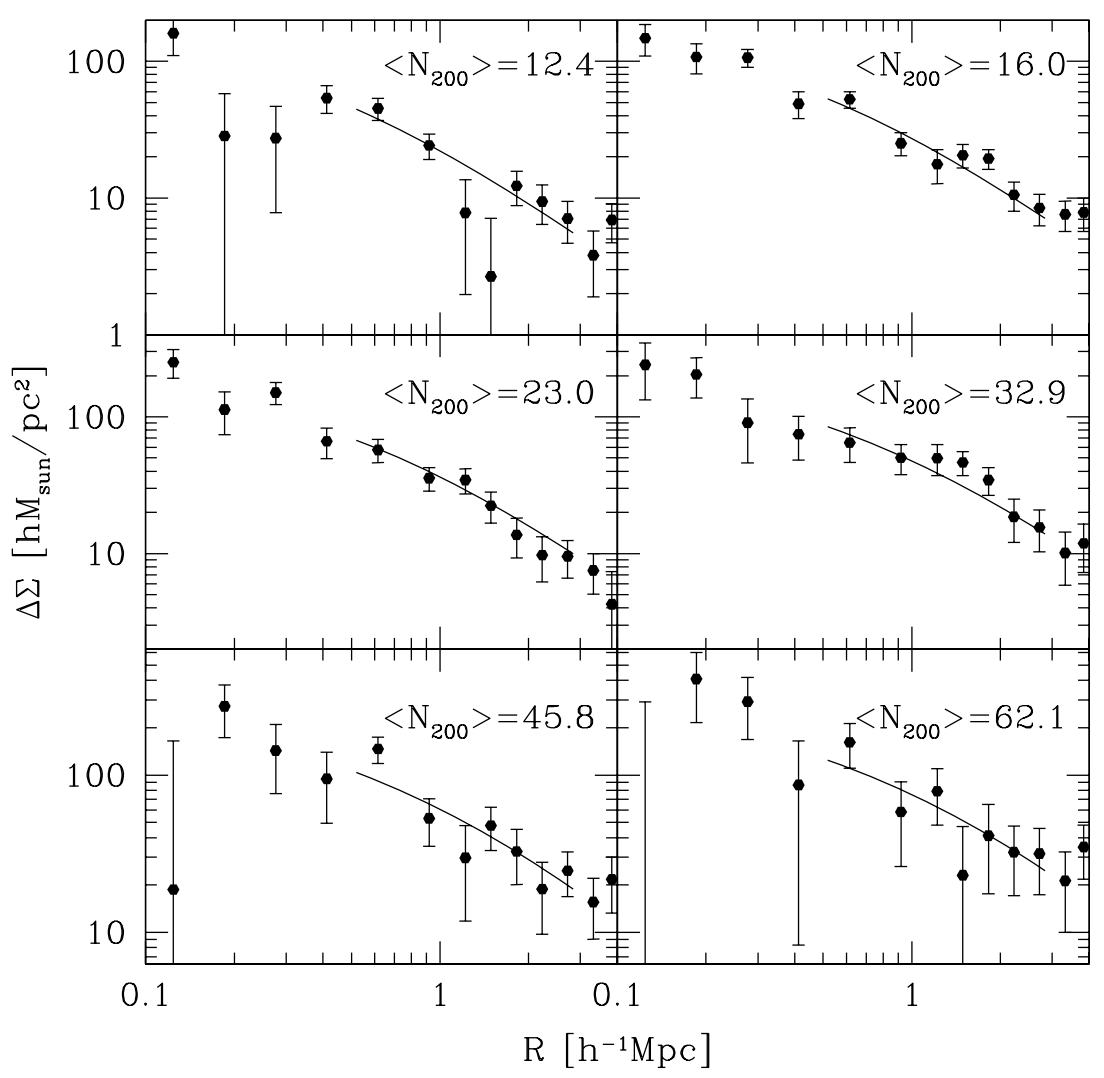

Figure 2. The lensing signal $\Delta \Sigma$ and model prediction for the best-fit model parameters (fit 2 in table 2), for the maxBCG sample split into six richness bins.

clearly provide a good fit to the data in all lens samples, as will also be evident in the $\chi^{2}$ values for the fits, discussed below.

\subsection{Description of fits}

Table 2 shows the best-fit parameters for the power law relations $c(M)$ and maxBCG $M\left(N_{200}\right)$, using several approaches to the fits as described below. The table includes information about the fit minimum radii and whether offsets of BCGs were accounted for in the fitting. It also shows the $\chi^{2}$ per degree of freedom, indicating that all fits shown provide a reasonable fit to the data. As will be discussed in detail later, fit 2 in that table is used for any plots that include a best-fit model, because the fit procedure represents the best possible trade-off between systematic error due to cluster centroiding errors and statistical error. Table 3 shows the best-fit masses for the two LRG lens samples and the five lower luminosity samples for fit 2 in table 2 .

The tables include the results for several types of fits with varying minimum radii for the maxBCG sample; these results were shown to test several possible issues. In all cases, the maximum fit radius for the maxBCG clusters was $3.0 h^{-1} \mathrm{Mpc}$; the fits for the LRGs used $0.1-2 h^{-1} \mathrm{Mpc}$; and the fits for the lower luminosity samples used $0.04-0.5 h^{-1} \mathrm{Mpc}$. The maximum fit radius was chosen so that the halo-halo term remains small compared to the one-halo term. When computing the halo-halo term we assume the cosmological 
Lower luminosity ellipticals (left) and spirals (right)

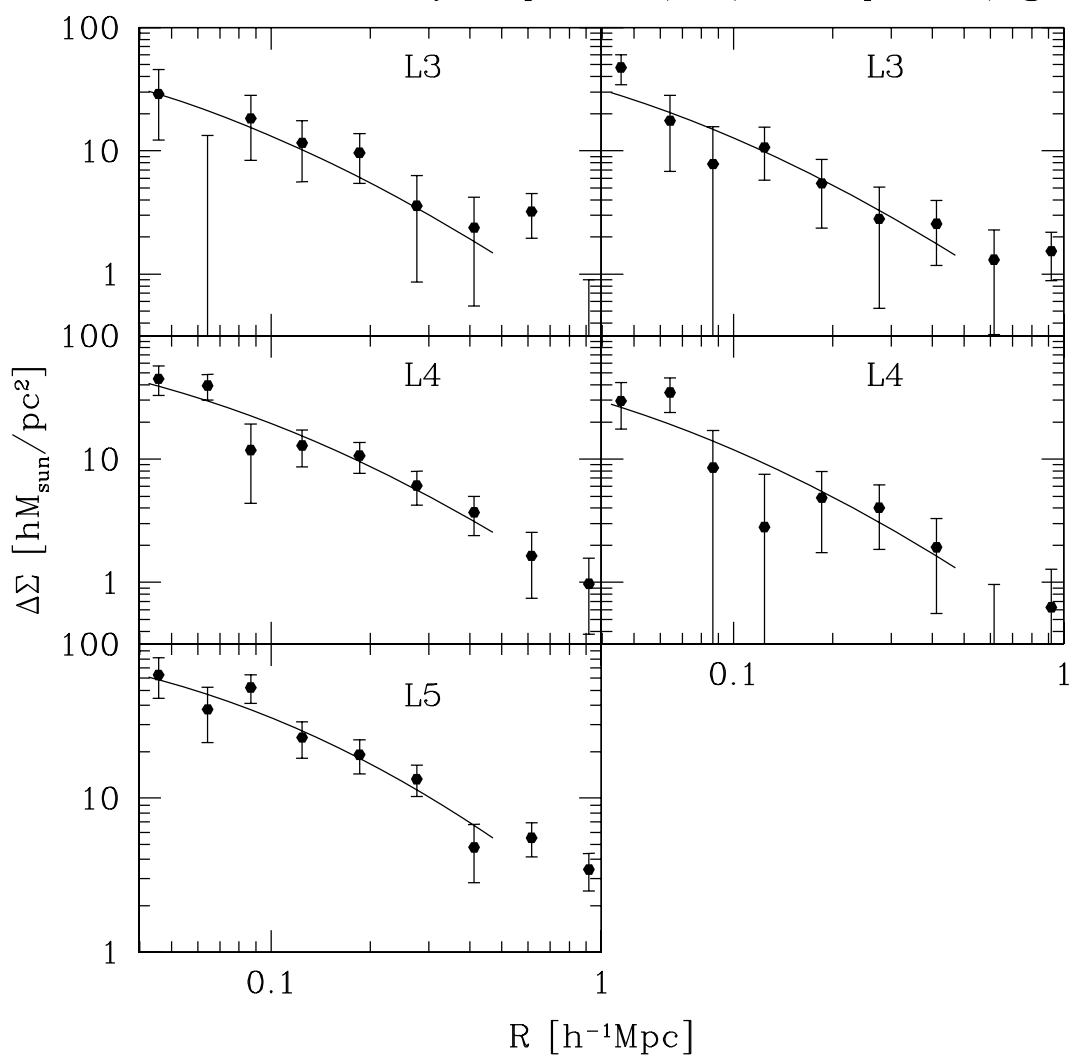

Figure 3. The lensing signal $\Delta \Sigma$ and model prediction for the best-fit model parameters (fit 2 in table 2), for the field low luminosity sample split into five morphology and luminosity bins (left column: ellipticals; right column: spirals).

model and redshift of these samples described in section 2.1. For example, for the lower mass LRG sample, a mass of $3 \times 10^{13} h^{-1} M_{\odot}$ gives a bias of 1.8. We compare this number against the results in [92], which gives a clustering amplitude for this sample relative to $L_{*}$ of 1.85. The highest richness maxBCG sample, approaching $M \sim 6 \times 10^{14} h^{-1} M_{\odot}$, has a bias of 5.5 in this model.

We justify our neglect of the stellar term by considering that a point mass at the origin gives a lensing signal $\Delta \Sigma=M_{\text {point }} /\left(\pi R^{2}\right)$. For the $L_{*}$ samples, typical stellar masses are a few $\times 10^{10} h^{-1} M_{\odot}$ and the minimum fit radius is $0.04 h^{-1} \mathrm{Mpc}$, which implies that the signal from the stellar component at that radius is subdominant compared to the observed signal of tens of $h M_{\odot} \mathrm{pc}^{-2}$. For the LRGs, the stellar mass is higher by factors of a few, but the minimum fit radius is 2.5 times higher, which again makes the stellar component subdominant on the scales used for the fit. Finally, for the maxBCG sample, given point masses of order $10^{12} h^{-1} M_{\odot}$ and at least $0.2 h^{-1} \mathrm{Mpc}$ for the minimum fit radius, it is difficult to arrange for the stellar term to be more than $5 \%$ of the predicted signal from the NFW dark matter halo.

3.1.1. Basic fits. We begin by discussing the basic fits 1-3, which use minimum fit radii of $0.2,0.5$, and $1 h^{-1} \mathrm{Mpc}$, without explicitly accounting for failure to properly 


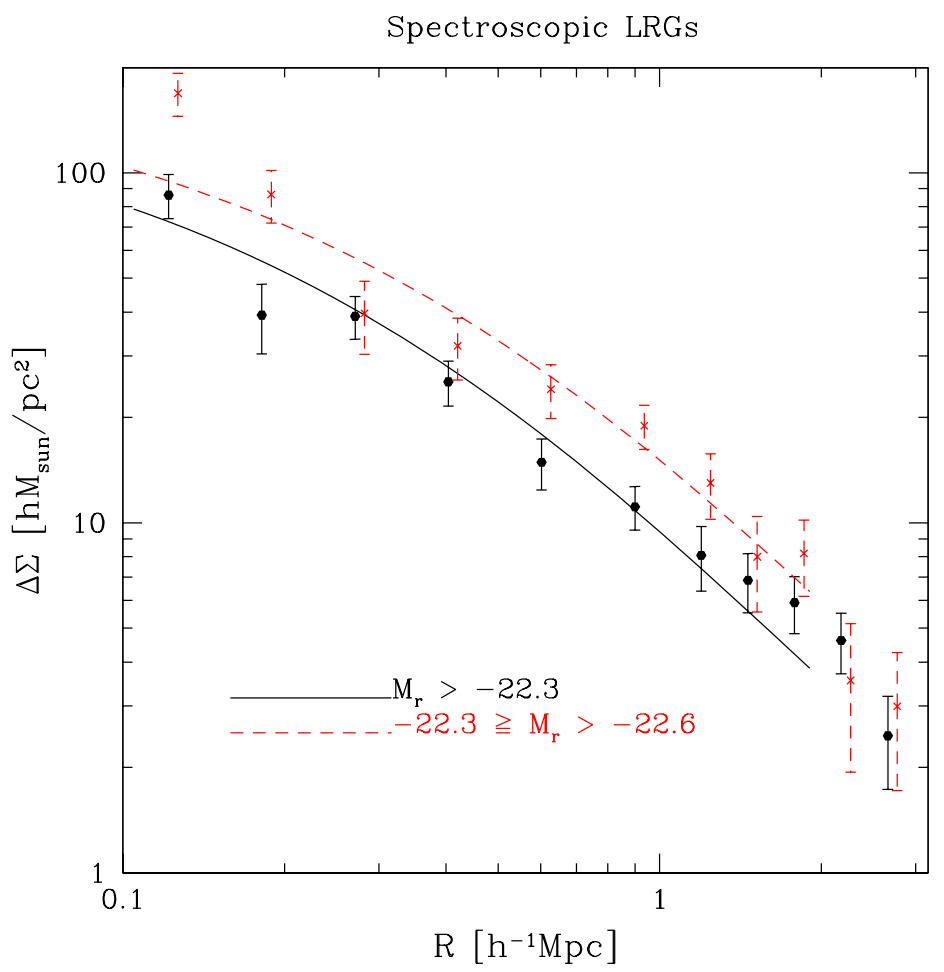

Figure 4. The lensing signal $\Delta \Sigma$ and model prediction for the best-fit model parameters, for the host LRG sample in two luminosity bins as labeled on the plot.

Table 2. Results of fits to lensing signal for several different minimum fit radii for the maxBCG sample split into six richness bins, with and without the prescription to account for BCG offsets described in section 2.1. Fits 1-6 have $\beta$ (the power law slope for the $c(M)$ relation) free; it is fixed to the theoretical value of 0.1 in fits $7-12$.

\begin{tabular}{|c|c|c|c|c|c|c|c|c|}
\hline Fit & $\begin{array}{l}R_{\min } \\
\left(h^{-1} \mathrm{Mpc}\right)\end{array}$ & Offsets & $c_{0}$ & $\beta$ & $\operatorname{Corr}\left(c_{0}, \beta\right)$ & $\begin{array}{l}M_{0} \\
\left(10^{14} h^{-1} M_{\odot}\right)\end{array}$ & $\gamma$ & $\chi^{2} / \nu$ \\
\hline 1 & 0.2 & No & $4.2 \pm 0.5$ & $0.16 \pm 0.07$ & -0.37 & $1.56 \pm 0.12$ & $1.20 \pm 0.14$ & $314.8 / 336$ \\
\hline 2 & 0.5 & No & $4.6 \pm 0.7$ & $0.13 \pm 0.07$ & -0.63 & $1.56 \pm 0.12$ & $1.15 \pm 0.14$ & $266.2 / 282$ \\
\hline 3 & 1.0 & No & $4.0 \pm 0.9$ & $0.18 \pm 0.09$ & -0.75 & $1.53 \pm 0.12$ & $1.23 \pm 0.18$ & $215.3 / 240$ \\
\hline 4 & 0.2 & Yes & $5.8 \pm 0.5$ & $0.03 \pm 0.07$ & -0.44 & $1.69 \pm 0.13$ & $1.07 \pm 0.15$ & $317.1 / 336$ \\
\hline 5 & 0.5 & Yes & $5.8 \pm 0.7$ & $0.03 \pm 0.07$ & -0.67 & $1.69 \pm 0.12$ & $1.08 \pm 0.14$ & $270.4 / 282$ \\
\hline 6 & 1.0 & Yes & $4.2 \pm 0.9$ & $0.16 \pm 0.09$ & -0.75 & $1.61 \pm 0.12$ & $1.22 \pm 0.18$ & $214.3 / 240$ \\
\hline 7 & 0.2 & No & $4.3 \pm 0.4$ & 0.1 & - & $1.53 \pm 0.11$ & $1.17 \pm 0.13$ & $315.9 / 337$ \\
\hline 8 & 0.5 & No & $4.8 \pm 0.6$ & 0.1 & - & $1.55 \pm 0.11$ & $1.14 \pm 0.13$ & $266.2 / 283$ \\
\hline 9 & 1.0 & No & $4.6 \pm 0.8$ & 0.1 & - & $1.50 \pm 0.11$ & $1.19 \pm 0.17$ & $215.7 / 241$ \\
\hline 10 & 0.2 & Yes & $5.5 \pm 0.4$ & 0.1 & - & $1.73 \pm 0.12$ & $1.10 \pm 0.14$ & $318.2 / 337$ \\
\hline 11 & 0.5 & Yes & $5.3 \pm 0.6$ & 0.1 & - & $1.72 \pm 0.11$ & $1.11 \pm 0.13$ & $271.3 / 283$ \\
\hline 12 & 1.0 & Yes & $4.7 \pm 0.8$ & 0.1 & - & $1.58 \pm 0.11$ & $1.19 \pm 0.17$ & $214.5 / 241$ \\
\hline
\end{tabular}


Table 3. Best-fit masses for LRGs and lower luminosity lens samples from the simultaneous fits to the lensing signal corresponding to fit 2 in table 2.

\begin{tabular}{ll}
\hline Sample & Mass $M_{200 b}\left(10^{12} h^{-1} M_{\odot}\right)$ \\
\hline LRG (fainter) & $30 \pm 3$ \\
LRG (brighter) & $56 \pm 5$ \\
Elliptical L3 & $1.0 \pm 0.4$ \\
Elliptical L4 & $1.8 \pm 0.4$ \\
Elliptical L5faint & $4.5 \pm 0.8$ \\
Spiral L3 & $0.9 \pm 0.3$ \\
Spiral L4 & $0.8 \pm 0.3$ \\
\hline
\end{tabular}

centroid BCGs. We first look for systematic effects due to BCG offsets by comparing the fit results with different minimum radii. The signature of BCG offsets would be a lower concentration for a lower minimum fit radius; we see no definitive sign of any such problem on the scales used here. For the case with minimum fit radius of $0.5 h^{-1} \mathrm{Mpc}$, which we adopt as a compromise between minimizing the systematic error due to this effect while maximizing the statistical constraining power, the concentration at $M_{200 b}=10^{14} h^{-1} M_{\odot}$ is $c_{0}=4.6 \pm 0.7$, with a power law scaling with mass of $-\beta=-0.13 \pm 0.07$. The mass normalization at $N_{200}=20$ is $M_{200 b}=(1.56 \pm 0.12) \times 10^{14} h^{-1} M_{\odot}$, with a scaling with richness of $1.15 \pm 0.14$. The $\chi^{2}$ of 266 for 282 degrees of freedom indicates that the fit is acceptable, as shown in figures 2-4. Our minimum maxBCG richness bin, at $\left\langle N_{200}\right\rangle=12.4$, therefore has mean mass and concentration in this model of $M_{200 b}=0.9 \times 10^{14} h^{-1} M_{\odot}$ and $c_{200 b}=4.7$.

The best-fit masses for the LRG and lower luminosity samples are shown in table 3 , and as we anticipated, the LRGs are in a lower mass range that does not overlap that of the maxBCG sample. The spectroscopic LRGs with $M_{0.0_{r}} \geq-22.6$ therefore predominantly trace group-scale halos, below the cluster scale masses of the maxBCG sample with $N_{200} \geq 12$. We also confirm our previous results [43] that, at fixed $r$-band luminosity, isolated $L_{*}$ spirals have a lower mass than isolated $L_{*}$ ellipticals. The signal detections are higher significance than the $\sigma_{M} / M$ values would indicate for L3: the error distributions determined using the bootstrap-resampled data sets are non-Gaussian and well separated from zero.

3.1.2. Fits with offsets. We next consider the other sets of fits in table 2. Fits 4-6 differ from 1-3 only in the inclusion of a prescription given in [45] to correct for the centroiding problem in the maxBCG catalog (with no change in the way the lower mass samples were handled). This prescription, derived from mock catalogs, is described in detail in [45]. In brief, it has a richness-dependent fraction of misidentified BCGs (from 30\% at low richness to $20 \%$ at high richness), and those that are misidentified have a Gaussian distribution of projected separation from the true centroid, with a scale radius of $0.42 h^{-1} \mathrm{Mpc}$.

The key point to consider is that if the model used to account for centroiding problems is correct, then the best-fit concentrations should be independent of the minimum scale used. We see that this is not the case: the concentrations are significantly elevated compared to previous results for minimum fit radii of 0.2 and $0.5 h^{-1} \mathrm{Mpc}$, and differ from the results with a minimum fit radius of $1 h^{-1} \mathrm{Mpc}$, for which (as one expects) the offsetting 
prescription does not significantly change the results given that the minimum fit radius is well outside the offset scale. Thus, we suggest that this particular offset procedure, which is determined using mock catalogs in [45], may in fact overcompensate for the true level of BCG offsets. This would not be too surprising, given that it depends sensitively on the contents of the mock catalogs, and is only applicable if they are a very true representation of the real world. At the high mass end, the comparison of maxBCG versus x-ray centroids in [44] can be used to evaluate the offset procedure derived from mock catalogs. For the derived $20 \%$ failure rate, with a Gaussian scale length of $0.42 h^{-1} \mathrm{Mpc}$ for the projected offset, we would expect 6.9 and $8.7 \%$ of the clusters to have centroids with projected offsets of $0.25-0.5$ and $0.5-1 h^{-1} \mathrm{Mpc}$, respectively. Out of the 87 clusters with x-ray matches within $1 h^{-1} \mathrm{Mpc}$, figure 14 in [44] shows 11 (12.6\%) and 4 (4.6\%) in those two ranges of projected offsets. While these numbers are not formally inconsistent with the expectations from the mocks (e.g., for the $0.5-1 h^{-1}$ Mpc offset range, the one-tailed $P(n \leq 4)=0.12$ ), had they been used to derive an empirical model for the offset, it would entail a smaller correction than that derived from the mocks at the scales which are most relevant for our adopted fit 2 . Since there may be uncertainty in the x-ray centroids for disturbed or merging clusters, even the lower estimate in [44] may be an overestimate.

The fact that [56] find similar levels of offsets for photometric LRGs from the centroids of cluster x-ray distributions does not contradict this conclusion: an examination of those results suggests that they only apply for masses above $\sim 5 \times 10^{14} h^{-1} M_{\odot}$ (after converting mass definitions), which essentially corresponds to the top two maxBCG bins considered here. Furthermore, the mean redshift for that study is higher than here, which implies different levels of photometric redshift errors and therefore different levels of BCG misidentification. Thus, it may not be applicable in detail here even for the top two bins. Finally, the use of higher mass clusters at higher redshifts should give a higher fraction of clusters that experienced recent mergers, so again we expect an overestimate of the effect for the full maxBCG sample. The true level of offsets in the four lower bins, which dominate the $c(M)$ fits, is poorly constrained from the real data.

3.1.3. Fits with fixed power law scaling. Next, to investigate the correlation between $c_{0}$ and $\beta$, we consider fits $7-12$, which are the same as $1-6$ except with a fixed $\beta=0.1$ (the theoretical value). While table 2 gives the formal coefficient of correlation between these parameters from the fit covariance matrix, it is instructive to explicitly fix $\beta=0.1$ to see the effect on $c_{0}$. As shown, there is some degeneracy between $c_{0}$ and $\beta$, but a comparison of e.g. fits 2 and 8 suggests that this degeneracy is not very strong for the fit that we have selected as our main result. Note that for fits 10-12, fixing $\beta$ has somewhat ameliorated the discrepancy between the fits using the offsetting procedure with different minimum scales. Nonetheless, the trend towards increasing $c$ with decreasing minimum scale is suggestive of possible overcompensation for the true level of the problem in reality.

As stated previously, we choose fit 2 as our most robust result, but the difference between this and the other fits suggests a systematic error that is comparable in size to the statistical error. Without better knowledge of the true level of offsets of the maxBCG from the cluster centroids (in the statistical sense), it is impossible to reduce this systematic error. However, for masses below a few $\times 10^{14} h^{-1} M_{\odot}$, where x-ray cluster data are difficult to obtain with sufficient resolution at these redshifts, there is no clear, simple way to observationally constrain these offsets at this time. 
3.1.4. Systematic tests of fitting procedure. Next, we discuss a few fits not included in the table that were designed to test for systematic errors. Recent work using the Millenium simulation [93] suggests that DM halos can be more properly described using the Einasto profile than the NFW profile,

$$
\rho(r)=\rho_{s} \mathrm{e}^{(-2 / \alpha)\left[\left(r / r_{s}\right)^{\alpha}-1\right]},
$$

where $\alpha$ has a weak mass dependence with a value around 0.15 . Thus, fits to NFW profiles in simulations can lead to different concentrations depending on the scale used for the fits. While the differences between Einasto and NFW profiles are most significant well within the scale radius, where we do not probe using weak lensing, we nonetheless test that our results are insensitive to the choice of Einasto versus NFW profiles. When doing a fit that is comparable to fit 2 but with NFW profiles replaced by Einasto profiles, we find the best-fit masses to be preserved, and the best-fit $c_{0}=4.5 \pm 0.7$ and $\beta=0.12 \pm 0.7$. The changes from fit 2 are well within the $1 \sigma$ error, so we conclude that possible errors in best-fit parameters due to the use of NFW rather than Einasto profiles are insignificant.

We also perform fit 2 using three broader bins in $N_{200}$ instead of the six narrow bins used for the main results. We find that the recovered $c(M)$ relation is virtually unchanged; the main parameter that varies is $\gamma$ in the $M\left(N_{200}\right)$ relation, which becomes shallower by $1.5 \sigma$. This result is as expected from [46]: the broad bins are most problematic at the highest mass end, where they reduce the best-fit mass. Since we are not fitting the bins for masses individually, but rather are fitting for a power law relation, the exponent of this relation is consequently reduced. Given the size of this shift, and the fact that our narrower bins used for the main analysis should contain mass distributions less than an order of magnitude wide, we do not ascribe significant systematic error in the concentration-mass relation to the default bin size.

Finally, because of potential centroiding systematics in the maxBCG lensing sample that should not be present for the lower luminosity or LRG samples, we performed the fits without the maxBCG samples entirely. In that case, we find that $\beta$ is quite poorly constrained, so we fix it to 0.1 and compare against fit 8 in table 2 , which also has $\beta=1$ and only differs in that it includes the maxBCG sample. In this case, we find the bestfit $c_{0}=4.7 \pm 0.7$, entirely consistent with the results in the table. This result suggests that our choice of minimum fit radius has minimized systematic error due to maxBCG centroiding errors to be well within the statistical error.

\subsection{Concentration-mass relation}

In figure 5 , we show the best-fit $c(M)$ relation from fit 2 , with a $1 \sigma$ error region defined by the fits to fifty bootstrap-resampled data sets. As shown, the concentration-mass relation is best constrained from $10^{13}-10^{14} h^{-1} M_{\odot}$, due to the interplay between higher mass increasing the lensing signal versus higher mass meaning a lower number density (and therefore higher measurement error). We emphasize that this is the $c(M)$ relation at $z \sim 0.22$, so in the simplest approximation of no mergers, the normalization at $z=0$ should be higher by about $20 \%$. As shown, the range dominated by the $\sim L_{*}$ samples $\left(10^{12} h^{-1} M_{\odot}\right)$ yields a concentration of $10 \pm 3$, as expected theoretically. At $6 \times 10^{14} h^{-1} M_{\odot}$, the top end of the maxBCG sample, the constraint is $4 \pm 1$. A constant concentrationmass relation is just barely permitted at the $2 \sigma$ level. The red points on the plot are the best-fit concentrations and masses for the individual lens samples when we fit for $c$ and 


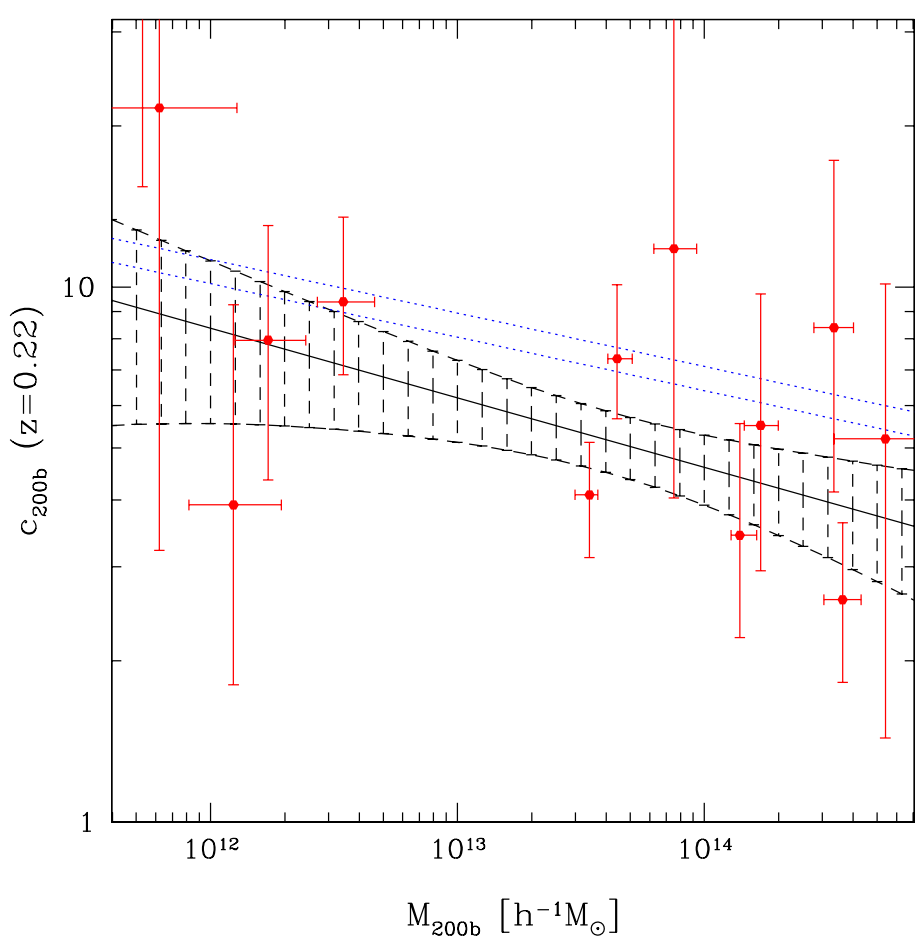

Figure 5. The best-fit $c(M)$ relation at $z=0.22$ with the $1 \sigma$ allowed region indicated. The red points with error bars show the best-fit masses and concentrations for each bin when we fit them individually, without requiring a power law $c(M)$ relation. The blue dotted lines show the predictions of [39] for our mass definition and redshift, for the WMAP1 (higher) and WMAP3 (lower) cosmologies. The prediction for the WMAP5 cosmology falls in between the two and is not shown here.

$M$ for each one without requiring a power law $c(M)$ relation. The consistency with this power law indicates that within the error bars, the $c(M)$ power law is indeed a good fit to the data.

\subsection{Comparison against previous observations}

We can compare these results to our previous lensing results based solely on the LRG sample [42]. In that case we found $c_{200 b}=5.2 \pm 0.6$ at the pivot mass of $\sim 5 \times 10^{13} h^{-1} M_{\odot}$, with weak constraints on the slope of the mass-concentration relation given the narrow mass range traced via LRG halos. This number is in good agreement with our fiducial value $c_{200 b}=4.7 \pm 0.7$ at $10^{14} h^{-1} M_{\odot}$, which gets increased by $10 \%$ when going to the lower LRG masses. The LRG sample is one of the three samples used here and we follow essentially the same analysis, so the agreement is to some extent expected.

Next we compare our results against the weak lensing determination of $c(M)$ in [45], which differs from ours in several notable points: (1) we use the maxBCG sample to cover the range of masses from 0.8 to $6 \times 10^{14} h^{-1} M_{\odot}$, whereas they use a proprietary version of the catalog that extends roughly 1.5 orders of magnitude lower in mass; (2) we include several additional mass tracers extending the halo mass range a factor of $\sim 10$ lower 
than in [45] with very different selection criteria; (3) we avoid scales that are affected significantly by BCG centroiding problems, rather than using a correction procedure derived from mock catalogs; and (4) the photometric redshifts that they use to determine source redshifts and therefore normalize the lensing signal suppress the lensing signal by $\sim 15-20 \%$ [91].

In their table 10, they show fit results for power law relations between mass and richness, and concentration and richness. We consider their result for $M_{180 b}$, which should differ from our results with $M_{200 b}$ by only a few per cent. At $N_{200}=20$, they find a bestfit mass of $1.2 \times 10^{14} h^{-1} M_{\odot}$. This number is lower than our result in table 2 by $22 \%$; however, there is a straightforward reason for this difference. Using a large spectroscopic training sample, we have recently shown that the photometric redshift algorithm used for sources in that work leads to a suppression of the lensing signal for these lens redshifts of $\sim 20 \%$ [91] and hence leads to about 30\% underestimation of mass. They also find a steeper scaling with richness, 1.3 instead of 1.15 as in our work. This difference can be explained by the fact that they use a much larger range of richnesses for the fit, $N_{200} \geq 1$ rather than $\geq 12$ as in our work. It is apparent from their figure 11 that if one restricts to $N_{200} \geq 12$, the best-fit power law should be shallower than the result for their full richness range, roughly consistent with our result.

We also compare against their results for concentration as a function of richness. They find $c_{180 b}=6.14$ at $M_{180 b}=1.2 \times 10^{14} h^{-1} M_{\odot}$, but as we argue above, their mass is underestimated, so this should really correspond to $M_{180 b}=1.5 \times 10^{14} h^{-1} M_{\odot}$. Rescaling, with $\beta=-0.1$, we find their value at $M_{200 b}=10^{14} h^{-1} M_{\odot}$ is $c_{200 b}=6.4 \pm 0.3$. The central value is in good agreement with the value that we find when following their procedure of correcting the halo center misidentification with mock catalogs, $c_{200 b}=5.8 \pm 0.7$. The statistical error is larger in our case because we do not use the small scale information. Note that because the mocks are likely not to be a completely realistic description of the effect, Johnston et al [45] attach a relatively large systematic error of $30 \%$ on top of the relatively small statistical error that they obtain. Instead, we trade statistical power for reduction of systematic error by using the fits from $0.5 h^{-1} \mathrm{Mpc}$, where the effects of halo center misidentification are less severe, in which case we find $c_{200 b}=4.6 \pm 0.7$ for our standard fit with $\beta=0.1$ and no offsets, and very similar values also for the fits from scales above $1 h^{-1} \mathrm{Mpc}$, either with or without offsets. We conclude that our result is relatively insensitive to the offsetting procedure, and while the results agree within the errors, our concentrations are significantly lower.

Our results can be compared to those from other methods that are used to determine the density profiles. Recent cluster x-ray and strong-weak lensing studies have found that the profile is consistent with the NFW model, but in many cases with a concentration that is higher than predicted by the concordance cosmology implied by WMAP [23]-[25], [29]. While some previous work concluded that this result implies a higher normalization cosmology, this interpretation may be premature. There are many alternative explanations that need to be explored, such as the use of information from scales below $100 h^{-1} \mathrm{kpc}$ in clusters, where baryons make a significant contribution to the density profile and tend to steepen the profile, therefore increasing the best-fit concentration. In addition, there are significant effects of triaxiality on the formation of arcs in the strong lensing regime. In the case of x-ray analysis, additional sources of pressure support may complicate the reconstruction based on hydrostatic equilibrium, since only the thermal pressure can be 
measured directly, while other sources of pressure, such as turbulence, cosmic rays or magnetic fields, cannot. In some cases, the gas may not be relaxed at all, and the hydrostatic equilibrium assumption is invalid. Another possibility is selection effects, since the dispersion of the concentration is large and correlates with x-rays or strong lensing selection such that only high concentration clusters near the mass threshold are in the sample [26]. It is important to compare the different tracers for consistency, and our analysis provides a complementary approach that can be compared against these more traditional analyses only once these other effects are understood in more detail.

\subsection{Comparison to simulations}

Next, we compare our concentration parameter fits to theoretical expectations. We show theoretical predictions on top of the data in figure 5, using the results from the Millenium simulations in [39] and [93]. Using a full sample, including both unrelaxed and relaxed halos, Neto et al $[39]$ find $c_{200 c}=4.67\left[M_{200 c} /\left(10^{14} h^{-1} M_{\odot}\right)\right]^{-0.11}$ (see also [94] and [95]). First, we move these $z=0$ results to our mean redshift of 0.22 , lowering the amplitude by $1 / 1.22$. Then, carefully converting both the mass and the concentration to account for the different halo definitions, we find the corresponding relation to be $c_{200 b}=7.1\left[M_{200 b} /\left(10^{14} h^{-1} M_{\odot}\right)\right]^{-0.1}$. This prediction is for the Millenium simulation cosmology with $\Omega_{\mathrm{m}}=0.25$ and $\sigma_{8}=0.9$; if we convert to $\sigma_{8}=0.82[96,97]$ by assuming that $c \propto M_{\mathrm{nl}}^{0.1}[2]$, then the predicted amplitude of this relation is reduced from 7.1 to 6.7. Finally, the results of $[93,98]$ suggest that the concentrations in [39] are too high by $10 \%$ at the high masses where we have the most statistical power, so this would bring the predicted value to 6.0 for the WMAP5 cosmology. Note that the scaling with mass no longer holds at the high mass end, where concentration becomes constant and is given roughly by $c_{200 b} \sim 5-6$. Simulations predict that this occurs at masses comparable to or slightly higher than our highest mass bin, so we will continue to use the power law scaling with mass in our analysis. To be more quantitative we plot the predictions for WMAP1 and WMAP3 cosmologies at $z=0.22$ for the $M_{200 b}$ mass definition on figure 5 together with the observational constraints. As shown, the results for the lower normalization cosmology are $\sim 2 \sigma$ above our measured concentration at $10^{14} h^{-1} M_{\odot}$ (fits 2 or 8 in table 2).

Typically the predicted profiles are derived from $N$-body simulations by fitting an NFW or Einasto profile to the 3D density distribution and averaging the profile obtained over all halos of a certain mass. There are many reasons why our observational procedure may differ from this. One is that we have both scatter in the mass-concentration relation and deviations from sphericity, both of which can change the mean profile of $2 \mathrm{D} \Delta \Sigma$ when compared to the average density in 3D. We also have scatter in the mass-richness relation, so that the assumption of a narrow mass distribution may be violated. When using the signal for central galaxies in the brightest luminosity bin in the simulations from [46], which incorporate both scatter in the mass-luminosity relationship and in the concentration-mass relationship, we found that the best-fit concentration can be $20 \%$ lower than if there is no scatter. However, the comparison to the expected concentration at the corresponding mass suggests that even without scatter, the concentration fits can be biased by up to $20 \%$, possibly due to deviations of the average profile from NFW or Einasto, which show up differently in $\Delta \Sigma(R)$ than in the spherical radial profile $\rho(r)$. 
Another comparison in [58] also found that the concentration derived from $\Delta \Sigma(R)$ can be either an underestimate (at low masses) relative to what is derived from the radial profile or an overestimate (at higher masses), but it is not clear how this result should be applied to our analysis since that analysis did not attempt to mimic our fitting procedure in detail.

Another uncertainty arises from the predicted values for concentration in existing simulations. Dolag et al [99] find values that are about 10-20\% higher than the values used above. It is not clear how worrisome this is, given that it was derived from only a handful of preselected massive clusters. A more concerning issue is the difference between relaxed halos versus all halos when fitting for concentrations. When Neto etal [39] use relaxed clusters only when fitting for concentration, they find about $10 \%$ higher values than for the full sample. Similarly, Macciò et al [94] find a large difference between the two, with relaxed clusters having typically $20 \%$ higher concentrations at these masses. On the other hand, analysis of relaxed clusters in [93] can be compared to the full analysis presented in [95] and the latter gives only a 5\% reduction in the values of concentrations at the halo masses around $10^{14} h^{-1} M_{\odot}$. Our sample consists of most or all halos above a certain mass threshold in a given volume; hence we should compare it to the full sample, which could bring the observed and predicted values into a better agreement.

The above discussion suggests that there is some theoretical uncertainty in the predicted values of concentrations, at a level of $20 \%$, that prevents us from making a more quantitative comparison to our results. This uncertainty could be reduced if exactly the same analysis used here on the real data is repeated on a large sample of simulated clusters, but doing so requires a large library of simulated galaxies and clusters from cosmological simulations with a volume comparable to or larger than that used in the actual data analysis (of the order of $\mathrm{Gpc}^{3}$ ) yet with high mass resolution, for which the next generation of simulations will be required, and is thus beyond the scope of this paper.

\subsection{Implications for shear-shear lensing}

Our results also have implications for the theoretical interpretation of shear-shear lensing. The weak lensing power spectrum quantifies galaxy distortions produced by lensing, which is sensitive to all matter in the universe, and as such it has long been advertised as being insensitive to astrophysical uncertainties present in other tracers such as galaxies. However, this argument relies on the assumption that the baryonic effects on the distribution of total matter can be understood. While earlier estimates found the effect to be small $[100,101]$, recent work based on simulations with gas cooling finds that this assumption may be invalid [102,103]. They find that the baryons cause a significant redistribution of matter within a halo, such that cooling and compression of baryons towards the center also makes the dark matter more concentrated. This redistribution can increase the concentration relative to the theoretical model predictions by up to $40 \%[103,104]$, and suggests that the matter profile is significantly redistributed well outside the inner region of the halo where gas has been transformed into stars.

Observationally, we find no evidence for such an increase in concentration. We find that concentrations are at the lower end of the range predicted by simulations even for the low normalization cosmology implied by WMAP3, and more so for the latest determinations of normalization and matter density $[96,97]$, which are already 
above the observations by $2 \sigma$. An increase in predicted concentrations by $40 \%$ would make the discrepancy more than $5 \sigma$, which is ruled out by our analysis. If halo center misidentification were considerably worse than estimated, then such a concentration enhancement would still be possible, but this would then be inconsistent with our analysis of halo concentration determination as a function of inner radius (see table 2), where we find no evidence of a systematic change in concentration with radius. It would also be inconsistent with our comparison between the analyses with and without displacements, for which we find the difference to be within the statistical error if the analysis excludes information below $0.5 h^{-1} \mathrm{Mpc}$. Thus, on scales larger than this, effects due to baryon cooling are likely to be small, so their effect on the existing shear-shear measurements can be neglected.

In the future, the statistical error of shear-shear autocorrelation measurements will be significantly reduced, so the baryonic effects may therefore become significant, but at the same time, the measurements of the halo profiles will improve as well. Thus, the effect can to some extent be corrected for by including the differences between the observed and predicted galaxy-galaxy and cluster-galaxy lensing profiles in the analysis. One approach to doing this is through the halo model analysis of the dark matter power spectrum [105]-[108], which has been shown to give a good agreement with the simulations [103]. A necessary requirement, however, is that our understanding of BCG halo center displacements improves, either through observations or via simulations.

\section{Conclusions}

We used a large sample of 170640 isolated spectroscopic galaxies, 38236 groups traced via spectroscopic LRGs and 13823 maxBCG clusters from SDSS, and applied a weak lensing analysis to determine their average masses and matter density profiles as a function of halo mass. We fit the lensing signal to an NFW or Einasto profile, excluding small scales to reduce the effects of baryons and misidentification of halo centers. The largest scale that we use in the fits is $3 h^{-1} \mathrm{Mpc}$ for clusters, where the large scale structure contribution is still small, but we account for it using a halo-halo term with a bias predicted by simulations. For galaxies, the largest scale that we use is $500 h^{-1} \mathrm{kpc}$, and for groups/LRGs, $1 h^{-1} \mathrm{Mpc}$.

Fitting the lensing signal to an NFW profile, we find that the cluster concentration weakly decreases with mass, $c=c_{0}\left(M / M_{0}\right)^{-\beta}$, with $\beta=0.13 \pm 0.07$ in good agreement with predictions from simulations. The mean concentration at $M_{200 b}=10^{14} h^{-1} M_{\odot}$ is $c_{200 b}=4.6 \pm 0.7(z=0.22)$. This value should be compared to the predicted value $\sim 6$ for the best-fit cosmological models $[96,97]$. The measured concentrations are below the predictions, although within $2 \sigma$. We find very little difference between NFW and Einasto profile in terms of the measured concentration.

While there appears to be a mild discrepancy between the predictions and observational constraints, there are significant uncertainties in the theoretical predictions that prevent us from robustly concluding whether there is a problem. One task for the future is to repeat exactly the same analysis as is done here on a representative sample of halos from cosmological simulations covering the mass range of observed halos. This should be possible in the near future as a new generation of large volume and high mass resolution $N$-body simulations becomes available, thus allowing for a more accurate calibration of the concentration-mass relation than is possible at the moment. 
However, to reduce the systematic uncertainty further we also need a better understanding of the displacement of BCGs from the halo center, which can be achieved either through observational studies [56] or through improved modeling in cosmological simulations [45]. In this paper we attempt to minimize it by using information outside the central region where we expect the effects from baryons in the central galaxy and from misestimation of the cluster center to be small. We see no evidence of systematic contamination in the sense that we find consistent results with and without accounting for the halo center misestimation, but we cannot completely exclude the possibility that there are residual effects at a level comparable to or below the statistical error. Even if we increase the measured concentrations by this amount, they do not exceed the predicted values, and thus we see no evidence of an enhancement in concentrations due to baryonic cooling predicted by some simulations $[102,103]$. This result bodes well for existing and future shear-shear weak lensing analyses, in that the baryonic effects are likely to be small and confined to small scales, and that by comparing theoretical and observed profiles as done here, these effects can be corrected for.

\section{Acknowledgments}

We thank the anonymous referee for useful comments on the submitted version of this paper. RM is supported by NASA through Hubble Fellowship grant No. HST-HF01199.02-A awarded by the Space Telescope Science Institute, which is operated by the Association of Universities for Research in Astronomy, Inc., for NASA, under contract NAS 5-26555. US is supported by the Packard Foundation, NSF CAREER-0132953 and Swiss National Foundation (grant number 200021-116696/1). CH is supported by DoE DE-FG03-92-ER40701.

Funding for the SDSS and SDSS-II has been provided by the Alfred P Sloan Foundation, the Participating Institutions, the National Science Foundation, the US Department of Energy, the National Aeronautics and Space Administration, the Japanese Monbukagakusho, the Max Planck Society, and the Higher Education Funding Council for England. The SDSS Web Site is http://www.sdss.org/.

The SDSS is managed by the Astrophysical Research Consortium for the Participating Institutions. The Participating Institutions are the American Museum of Natural History, Astrophysical Institute Potsdam, University of Basel, University of Cambridge, Case Western Reserve University, University of Chicago, Drexel University, Fermilab, the Institute for Advanced Study, the Japan Participation Group, Johns Hopkins University, the Joint Institute for Nuclear Astrophysics, the Kavli Institute for Particle Astrophysics and Cosmology, the Korean Scientist Group, the Chinese Academy of Sciences (LAMOST), Los Alamos National Laboratory, the Max Planck Institute for Astronomy (MPIA), the Max Planck Institute for Astrophysics (MPA), New Mexico State University, Ohio State University, University of Pittsburgh, University of Portsmouth, Princeton University, the United States Naval Observatory, and the University of Washington.

\section{References}

[1] Avila-Reese V, Firmani C, Klypin A and Kravtsov A V, 1999 Mon. Not. R. Astron. Soc. 310527 [astro-ph/9906260]

[2] Bullock J S, Kolatt T S, Sigad Y, Somerville R S, Kravtsov A V, Klypin A A, Primack J R and Dekel A, 2001 Mon. Not. R. Astron. Soc. 321559 
[3] Diemand J, Zemp M, Moore B, Stadel J and Carollo C M, 2005 Mon. Not. R. Astron. Soc. 364 665 [astro-ph/0504215]

[4] Fukushige T and Makino J, 1997 Astrophys. J. Lett. 477 L9 [astro-ph/9610005]

[5] Fukushige T and Makino J, 2001 Astrophys. J. 557533 [SPIRES] [astro-ph/0008104]

[6] Fukushige T and Makino J, 2003 Astrophys. J. 588674 [SPIRES]

[7] Ghigna S, Moore B, Governato F, Lake G, Quinn T and Stadel J, 2000 Astrophys. J. 544616 [SPIRES]

[8] Jing Y P and Suto Y, 2000 Astrophys. J. Lett. 529 L69

[9] Klypin A, Kravtsov A V, Bullock J S and Primack J R, 2001 Astrophys. J. 554903 [SPIRES]

[10] Kravtsov A V, Klypin A A and Khokhlov A M, 1997 Astrophys. J. Suppl. 11173

[11] Moore B, Governato F, Quinn T, Stadel J and Lake G, 1998 Astrophys. J. Lett. 499 L5

[12] Navarro J F, Frenk C S and White S D M, 1996 Astrophys. J. 462563 [SPIRES]

[13] Tasitsiomi A, Kravtsov A V, Gottlöber S and Klypin A A, 2004 Astrophys. J. 607125 [SPIRES] [astro-ph/0311062]

[14] Wechsler R H, Bullock J S, Primack J R, Kravtsov A V and Dekel A, 2002 Astrophys. J. 56852 [SPIRES] [astro-ph/0108151]

[15] Zhao D H, Jing Y P, Mo H J and Börner G, 2003 Astrophys. J. Lett. 597 L9 [astro-ph/0309375]

[16] Biviano A and Girardi M, 2003 Astrophys. J. 585205 [SPIRES] [astro-ph/0301439]

[17] Diaferio A, Geller M J and Rines K J, 2005 Astrophys. J. Lett. 628 L97 [astro-ph/0506560]

[18] Katgert P, Biviano A and Mazure A, 2004 Astrophys. J. 600657 [SPIRES] [astro-ph/0310060]

[19] Rines K, Geller M J, Kurtz M J and Diaferio A, 2003 Astron. J. 1262152 [SPIRES] [astro-ph/0306538]

[20] Rines K and Diaferio A, 2006 Astron. J. 1321275 [SPIRES] [astro-ph/0602032]

[21] Salucci P, Lapi A, Tonini C, Gentile G, Yegorova I and Klein U, 2007 Mon. Not. R. Astron. Soc. 37841 [astro-ph/0703115]

[22] Schuecker P, Finoguenov A, Miniati F, Böhringer H and Briel U G, 2004 Astron. Astrophys. 426387 [SPIRES] [astro-ph/0404132]

[23] Buote D A, Gastaldello F, Humphrey P J, Zappacosta L, Bullock J S, Brighenti F and Mathews W G, 2007 Astrophys. J. 664123 [SPIRES] [astro-ph/0610135]

[24] Schmidt R W and Allen S W, 2007 Mon. Not. R. Astron. Soc. 379209 [astro-ph/0610038]

[25] Vikhlinin A, Kravtsov A, Forman W, Jones C, Markevitch M, Murray S S and Van Speybroeck L, 2006 Astrophys. J. 640691 [SPIRES] [astro-ph/0507092]

[26] Fedeli C, Bartelmann M, Meneghetti M and Moscardini L, 2007 Astron. Astrophys. 473715 [SPIRES] [0705.3169]

[27] Bradač M, Clowe D, Gonzalez A H, Marshall P, Forman W, Jones C, Markevitch M, Randall S, Schrabback T and Zaritsky D, 2006 Astrophys. J. 652937 [SPIRES] [astro-ph/0608408]

[28] Broadhurst T et al, 2005 Astrophys. J. 62153 [SPIRES] [astro-ph/0409132]

[29] Comerford J M and Natarajan P, 2007 Mon. Not. R. Astron. Soc. 379190 [astro-ph/0703126]

[30] Sand D J, Treu T, Smith G P and Ellis R S, 2004 Astrophys. J. 60488 [SPIRES] [astro-ph/0310703]

[31] Zekser K C, White R L, Broadhurst T J, Benítez N, Ford H C, Illingworth G D, Blakeslee J P, Postman M, Jee M J and Coe D A, 2006 Astrophys. J. 640639 [SPIRES]

[32] Limousin M et al, 2007 Astrophys. J. 668643 [SPIRES] [astro-ph/0612165]

[33] Meneghetti M, Bartelmann M, Jenkins A and Frenk C, 2007 Mon. Not. R. Astron. Soc. 381171

[34] Hoekstra H, 2007 Mon. Not. R. Astron. Soc. 379317 [0705.0358]

[35] Pedersen K and Dahle H, 2007 Astrophys. J. 66726 [SPIRES] [astro-ph/0603260]

[36] Clowe D, Bradač M, Gonzalez A H, Markevitch M, Randall S W, Jones C and Zaritsky D, 2006 Astrophys. J. Lett. 648 L109 [astro-ph/0608407]

[37] Metzler C A, White M and Loken C, 2001 Astrophys. J. 547560 [SPIRES]

[38] Hoekstra H, 2003 Mon. Not. R. Astron. Soc. 3391155 [astro-ph/0208351]

[39] Neto A F, Gao L, Bett P, Cole S, Navarro J F, Frenk C S, White S D M, Springel V and Jenkins A, 2007 Mon. Not. R. Astron. Soc. 3811450 [0706.2919]

[40] Dahle H, Hannestad S and Sommer-Larsen J, 2003 Astrophys. J. Lett. 588 L73 [astro-ph/0206455]

[41] Sheldon E S et al, 2001 Astrophys. J. 554881 [SPIRES]

[42] Mandelbaum R, Seljak U, Cool R J, Blanton M, Hirata C M and Brinkmann J, 2006 Mon. Not. R. Astron. Soc. 372758 [astro-ph/0605476]

[43] Mandelbaum R, Seljak U, Kauffmann G, Hirata C M and Brinkmann J, 2006 Mon. Not. R. Astron. Soc. 368715 [astro-ph/0511164]

[44] Koester B P et al, 2007 Astrophys. J. 660239 [SPIRES] [astro-ph/0701265]

[45] Johnston D E, Sheldon E S, Wechsler R H, Rozo E, Koester B P, Frieman J A, McKay T A, Evrard A E, Becker M R and Annis J, 2007 Preprint 0709.1159 
[46] Mandelbaum R, Tasitsiomi A, Seljak U, Kravtsov A V and Wechsler R H, 2005 Mon. Not. R. Astron. Soc. 3621451 [astro-ph/0410711]

[47] Seljak U and Warren M S, 2004 Mon. Not. R. Astron. Soc. 355129 [astro-ph/0403698]

[48] Sheth R K and Tormen G, 1999 Mon. Not. R. Astron. Soc. 308119

[49] Eke V R, Navarro J F and Steinmetz M, 2001 Astrophys. J. 554114 [SPIRES]

[50] Blumenthal G R, Faber S M, Flores R and Primack J R, 1986 Astrophys. J. 30127 [SPIRES]

[51] Gnedin O Y, Kravtsov A V, Klypin A A and Nagai D, 2004 Astrophys. J. 61616 [SPIRES] [astro-ph/0406247]

[52] Naab T, Johansson P H, Ostriker J P and Efstathiou G, 2007 Astrophys. J. 658710 [SPIRES] [astro-ph/0512235]

[53] Koester B P, McKay T A, Annis J, Wechsler R H, Evrard A E, Rozo E, Bleem L, Sheldon E S and Johnston D, 2007 Astrophys. J. 660221 [SPIRES] [astro-ph/0701268]

[54] van den Bosch F C, Weinmann S M, Yang X, Mo H J, Li C and Jing Y P, 2005 Mon. Not. R. Astron. Soc. $\mathbf{3 6 1} 1203$ [astro-ph/0502466]

[55] Bildfell C, Hoekstra H, Babul A and Mahdavi A, 2008 Preprint 0802.2712

[56] Ho S, Lin Y-T, Spergel D and Hirata C M, 2007 Preprint 0706.0727

[57] Guzik J and Seljak U, 2002 Mon. Not. R. Astron. Soc. 335311

[58] Yang X, Mo H J, van den Bosch F C, Jing Y P, Weinmann S M and Meneghetti M, 2006 Mon. Not. R. Astron. Soc. 3731159 [astro-ph/0607552]

[59] York D G et al, 2000 Astron. J. 1201579 [SPIRES]

[60] Eisenstein D J et al, 2001 Astron. J. 1222267 [SPIRES]

[61] Richards G T et al, 2002 Astron. J. 1232945 [SPIRES]

[62] Strauss M A et al, 2002 Astron. J. 1241810 [SPIRES]

[63] Hogg D W, Finkbeiner D P, Schlegel D J and Gunn J E, 2001 Astron. J. 1222129 [SPIRES]

[64] Ivezić Ž et al, 2004 Astron. Nachrichten $\mathbf{3 2 5} 583$

[65] Fukugita M, Ichikawa T, Gunn J E, Doi M, Shimasaku K and Schneider D P, 1996 Astron. J. 1111748 [SPIRES]

[66] Smith J A et al, 2002 Astron. J. 1232121 [SPIRES]

[67] Gunn J E et al, 1998 Astron. J. 1163040 [SPIRES]

[68] Blanton M R, Lin H, Lupton R H, Maley F M, Young N, Zehavi I and Loveday J, 2003 Astron. J. 1252276 [SPIRES]

[69] Gunn J E et al, 2006 Astron. J. 1312332 [SPIRES] [astro-ph/0602326]

[70] Lupton R H, Gunn J E, Ivezić Z, Knapp G R, Kent S and Yasuda N, 2001 Astronomical Data Analysis Software and Systems X (ASP Conf. Ser. vol 238) (San Francisco, CA: Astronomical Society of the Pacific) p 269

[71] Pier J R, Munn J A, Hindsley R B, Hennessy G S, Kent S M, Lupton R H and Ivezić Ž, 2003 Astron. J. 1251559 [SPIRES]

[72] Tucker D L et al, 2006 Astron. Nachrichten 327821 [astro-ph/0608575]

[73] Abazajian K et al, 2003 Astron. J. 1262081 [SPIRES]

[74] Abazajian K et al, 2004 Astron. J. 128502 [SPIRES]

[75] Abazajian K et al, 2005 Astron. J. 1291755 [SPIRES]

[76] Adelman-McCarthy J K et al, 2006 Astrophys. J. Suppl. 16238 [astro-ph/0507711]

[77] Finkbeiner D P et al, 2004 Astron. J. 1282577 [SPIRES]

[78] Stoughton C et al, 2002 Astron. J. 123485 [SPIRES]

[79] Adelman-McCarthy J K et al, 2007 Astrophys. J. Suppl. 172634 [0707.3380]

[80] Adelman-McCarthy J K et al, 2008 Astrophys. J. Suppl. 175297 [0707.3413]

[81] Gladders M D and Yee H K C, 2000 Astron. J. 1202148 [SPIRES]

[82] Becker M R et al, 2007 Astrophys. J. 669905 [SPIRES] [0704.3614]

[83] Sheldon E S et al, 2007 Preprint 0709.1153

[84] Reyes R, Mandelbaum R, Hirata C M, Bahcall N and Seljak U, 2008 Preprint 0802.2365

[85] Blanton M R et al, 2005 Astron. J. 1292562 [SPIRES] [astro-ph/0410166]

[86] Schlegel D J, Finkbeiner D P and Davis M, 1998 Astrophys. J. 500525 [SPIRES]

[87] Bruzual G and Charlot S, 2003 Mon. Not. R. Astron. Soc. 3441000

[88] Blanton M R, Brinkmann J, Csabai I, Doi M, Eisenstein D, Fukugita M, Gunn J E, Hogg D W and Schlegel D J, 2003 Astron. J. 1252348 [SPIRES]

[89] Mandelbaum R, Hirata C M, Seljak U, Guzik J, Padmanabhan N, Blake C, Blanton M R, Lupton R and Brinkmann J, 2005 Mon. Not. R. Astron. Soc. 3611287 [astro-ph/0501201]

[90] Hirata C and Seljak U, 2003 Mon. Not. R. Astron. Soc. 343459 
[91] Mandelbaum R et al, 2008 Mon. Not. R. Astron. Soc. 386781 [0709.1692]

[92] Zehavi I, Eisenstein D J, Nichol R C, Blanton M R, Hogg D W, Brinkmann J, Loveday J, Meiksin A, Schneider D P and Tegmark M, 2005 Astrophys. J. 62122 [SPIRES] [astro-ph/0411557]

[93] Gao L, Navarro J F, Cole S, Frenk C S, White S D M, Springel V, Jenkins A and Neto A F, 2008 Mon. Not. R. Astron. Soc. 387536 [0711.0746]

[94] Macciò A V, Dutton A A, van den Bosch F C, Moore B, Potter D and Stadel J, 2007 Mon. Not. R. Astron. Soc. 37855 [astro-ph/0608157]

[95] Hayashi E and White S D M, 2008 Mon. Not. R. Astron. Soc. 3882 [0709.3933]

[96] Seljak U, Slosar A and McDonald P, 2006 J. Cosmol. Astropart. Phys. JCAP10(2006)14 [SPIRES] [astro-ph/0604335]

[97] Komatsu E et al, 2008 Preprint 0803.0547

[98] Duffy A R, Schaye J, Kay S T and Dalla Vecchia C, 2008 Preprint 0804.2486

[99] Dolag K, Bartelmann M, Perrotta F, Baccigalupi C, Moscardini L, Meneghetti M and Tormen G, 2004 Astron. Astrophys. 416853 [SPIRES] [astro-ph/0309771]

[100] White M, 2004 Astropart. Phys. 22211 [SPIRES]

[101] Zhan H and Knox L, 2004 Astrophys. J. Lett. 616 L75 [astro-ph/0409198]

[102] Jing Y P, Zhang P, Lin W P, Gao L and Springel V, 2006 Astrophys. J. Lett. 640 L119 [astro-ph/0512426]

[103] Rudd D H, Zentner A R and Kravtsov A V, 2008 Astrophys. J. 67219 [SPIRES] [astro-ph/0703741]

[104] Zentner A R, Rudd D H and Hu W, 2008 Phys. Rev. D 77043507 [SPIRES] [0709.4029]

[105] Peacock J A and Smith R E, 2000 Mon. Not. R. Astron. Soc. 3181144

[106] Ma C and Fry J N, 2000 Astrophys. J. 543503 [SPIRES]

[107] Seljak U, 2000 Mon. Not. R. Astron. Soc. 318203

[108] Scoccimarro R, Sheth R K, Hui L and Jain B, 2001 Astrophys. J. 54620 [SPIRES] 